\title{
Vainshtein mechanism in massive gravity nonlinear sigma models
}

\author{
Katsuki Aoki ${ }^{1, *}$ and Shuntaro Mizuno ${ }^{2, \dagger}$ \\ ${ }^{1}$ Department of Physics, Waseda University, Shinjuku, Tokyo 169-8555, Japan \\ ${ }^{2}$ Waseda Institute for Advanced Study, Waseda University, Shinjuku, Tokyo 169-8050, Japan
}

(Received 8 July 2016; published 20 September 2016)

\begin{abstract}
We study the stability of the Vainshtein screening solution of massive gravity/bigravity based on the massive nonlinear sigma model as the effective action inside the Vainshtein radius. The effective action is obtained by taking the $\Lambda_{2}$ decoupling limit around a curved spacetime. First we derive a general consequence that any Ricci flat Vainshtein screening solution is unstable when we take into account the excitation of the scalar graviton only. This instability suggests that the nonlinear excitation of the scalar graviton is not sufficient to obtain a successful Vainshtein screening in massive gravity/bigravity. Then to see the role of the excitation of the vector graviton, we study perturbations around the static and spherically symmetric solution obtained in bigravity explicitly. As a result, we find that linear excitations of the vector graviton cannot be helpful and the solution still suffers from a ghost and/or a gradient instability for any parameters of the theory for this background.
\end{abstract}

DOI: 10.1103/PhysRevD.94.064054

\section{INTRODUCTION}

The current acceleration of the Universe is one of the biggest problems in modern cosmology. It has been proposed to explain this acceleration with a modification of the theory of gravity from general relativity (GR) in the infrared regime (see [1-3] for reviews). However, modification of gravity is strongly constrained by Solar System tests of gravity that agree with the predictions of GR. Hence the effect of the modification of gravity must be screened at the Solar System level. One natural theory with such a screening mechanism is the massive gravity with a tiny graviton mass. In short scales, the massive graviton may behave as a massless graviton; thus one expects that the prediction of GR can be recovered. However, linear massive gravity [4] cannot be restored to the linearized GR because of the nonvanishing fifth force mediated by the scalar graviton $[5,6]$. Vainshtein then proposed that the linear approximation is no longer valid inside the Vainshtein radius and that the fifth force could be screened by nonlinear interactions, called the Vainshtein mechanism [7].

The Vainshtein mechanism has an important role not only in massive gravity but also in some classes of scalartensor theories that have nonlinear interactions [8-15]. However, because of their nonlinear interactions, a general analysis of the Vainshtein mechanism is quite complicated. Hence to discuss the Vainshtein mechanism, it should be useful to construct an effective theory from an original theory and discuss the Vainshtein mechanism based on the effective theory [16-19].

In this paper, we discuss an effective theory for the Vainshtein mechanism from nonlinear massive gravity

\footnotetext{
kkatsuki-a12@gravity.phys.waseda.ac.jp

† shuntaro.mizuno@aoni.waseda.jp
}

[20,21] and bigravity [22] (see [23-26] for reviews). The Vainshtein mechanism in massive gravity/bigravity has been discussed in [27-47]. It is known that nonlinear massive gravity can be reduced into the scalar-tensor theory with Galileon interactions by taking the $\Lambda_{3}$ decoupling limit when the vector graviton is not excited, in which there still exist scalar-tensor interactions [20,48] (see also $[49,50])$. On the other hand, one can take a more direct decoupling limit called the $\Lambda_{2}$ decoupling limit [51,52], in which the tensor fluctuations are decoupled from the scalar and vector gravitons and the effective action for the scalar and vector gravitons is given by the massive gravity nonlinear sigma model. The papers [51,52] discussed the $\Lambda_{2}$ decoupling limit around Minkowski spacetime. Contrary to this, in the present paper, we discuss the $\Lambda_{2}$ decoupling limit around a curved spacetime and obtain an effective theory inside the Vainshtein radius. Indeed the solution obtained by the effective theory gives an approximate solution inside the Vainshtein radius in bigravity theory [47]. Then we study the stability of the Vainshtein screening solution based on the effective theory.

The paper is organized as follows. We derive the effective theory for the Vainshtein mechanism from massive gravity/bigravity in Sec. II. In Sec. III we then study the dynamics of the scalar graviton around general backgrounds and find that a Ricci flat spacetime generally suffers from a ghost and/or a gradient instability. The instability is found when we ignore the vector graviton; however, perturbations of scalar and vector gravitons are coupled to each other, in general. Hence to complete the stability analysis of the solution, we should include perturbations of the vector graviton, which is studied in Sec. IV. We explicitly show that the static and spherically symmetric solution is unstable. We give a summary and some discussions in Sec. V. 


\section{EFFECTIVE ACTION INSIDE VAINSHTEIN RADIUS}

In this section, we show that the massive gravity nonlinear sigma model gives an effective theory of the vector and scalar gravitons inside the Vainshtein radius for general massive gravity/bigravity as long as we have the Vainshtein screening solutions. Let us start with the action [22] given by

$$
\begin{aligned}
S= & \frac{1}{2 \kappa_{g}^{2}} \int d^{4} x \sqrt{-g} R(g)+\frac{1}{2 \kappa_{f}^{2}} \int d^{4} x \sqrt{-f} \mathcal{R}(f) \\
& -\frac{m^{2}}{\kappa^{2}} \int d^{4} x \sqrt{-g} \sum_{n=2}^{4} c_{n} \mathcal{U}_{n}(\gamma)+S^{[\mathrm{m}]},
\end{aligned}
$$

where $g_{\mu \nu}$ and $f_{\mu \nu}$ are two dynamical metrics, and $R(g)$ and $\mathcal{R}(f)$ are their Ricci scalars. The interactions between the two metrics are given by

$$
\begin{aligned}
& \mathcal{U}_{2}(\mathcal{K})=-\frac{1}{4} \epsilon_{\mu \nu \rho \sigma} \epsilon^{\alpha \beta \rho \sigma} \mathcal{K}^{\mu}{ }_{\alpha} \mathcal{K}^{\nu}{ }_{\beta}, \\
& \mathcal{U}_{3}(\mathcal{K})=-\frac{1}{3} \epsilon_{\mu \nu \rho \sigma} \epsilon^{\alpha \beta \gamma \sigma} \mathcal{K}^{\mu}{ }_{\alpha} \mathcal{K}^{\nu}{ }_{\beta} \mathcal{K}^{\rho}{ }_{\gamma}, \\
& \mathcal{U}_{4}(\mathcal{K})=-\frac{1}{4} \epsilon_{\mu \nu \rho \sigma} \epsilon^{\alpha \beta \gamma \delta} \mathcal{K}^{\mu}{ }_{\alpha} \mathcal{K}^{\nu}{ }_{\beta} \mathcal{K}^{\rho}{ }_{\gamma} \mathcal{K}^{\sigma}{ }_{\delta},
\end{aligned}
$$

with $\mathcal{K}^{\mu}{ }_{\nu}=\delta^{\mu}{ }_{\nu}-\gamma^{\mu}{ }_{\nu}$ and $\gamma^{\mu}{ }_{\nu}$ is defined by

$$
\gamma_{\rho}^{\mu} \gamma_{\nu}^{\rho}=g^{\mu \rho} f_{\rho \nu}
$$

The parameters $\kappa_{g}^{2}=8 \pi G$ and $\kappa_{f}^{2}=8 \pi \mathcal{G}$ are the corresponding gravitational constants, while $\kappa$ is defined by $\kappa^{2}=\kappa_{g}^{2}+\kappa_{f}^{2}$.

The matter action is assumed such that a matter field can couple with either $g_{\mu \nu}$ or $f_{\mu \nu}$ :

$$
S^{[\mathrm{m}]}=S_{g}^{[\mathrm{m}]}\left(\psi_{g}, g\right)+S_{f}^{[\mathrm{m}]}\left(\psi_{f}, f\right) .
$$

Notice that our setup of bigravity is so general that it includes ghost-free massive gravity [20,21] as a special case. It is obtained by fixing $f$-spacetime as the Minkowski one with the limit $\kappa_{f} \rightarrow 0$ [53].

In what follows we set $c_{2}=-1$, which guarantees that the parameter $m$ corresponds to the graviton mass propagating on the Minkowski vacuum. ${ }^{1}$ We also introduce four Stuieckelberg fields $\phi^{a}(x)$, with which the metric $f_{\mu \nu}$ can be written by

$$
f_{\mu \nu}(x)=\frac{\partial \phi^{a}}{\partial x^{\mu}} \frac{\partial \phi^{b}}{\partial x^{\nu}} f_{a b}\left(\phi^{a}(x)\right),
$$

to see the dynamics of the vector and scalar gravitons in a clear way.

\footnotetext{
${ }^{1}$ In general, the ghost-free interactions include a constant term $\mathcal{U}_{0}$ and a tadpole term $\mathcal{U}_{1}(\mathcal{K})$. Although we drop them in this paper, just for simplicity, including these terms does not change our main conclusion.
}

It was shown in Refs. [51,52] that in the case of ghostfree massive gravity, about nontrivial vacua

$$
g_{\mu \nu}=\eta_{\mu \nu}+\mathcal{O}\left(m^{2}\right), \quad \phi^{a}=\bar{\phi}^{a}(x) \neq x^{a},
$$

and in the $\Lambda_{2}$ decoupling limit, given by

$$
m, \kappa_{g}, \kappa_{f} \rightarrow 0, \quad \Lambda_{2} \equiv \sqrt{m / \kappa_{g}} \text { : finite }
$$

an interesting effective theory for $\phi^{a}$, the so-called massive gravity nonlinear sigma model, described by the following action,

$$
S_{\mathrm{MG}-\mathrm{NLS}}^{(0)}=-\Lambda_{2}^{4} \int d^{4} x \sqrt{-\eta} \sum_{n=2}^{4} c_{n} \mathcal{U}_{n}\left(\gamma_{\mathrm{NLS}}\right),
$$

$\gamma_{\mathrm{NLS}}{ }_{\rho}^{\mu} \gamma_{\mathrm{NLS}}{ }_{\nu}=\eta^{\mu \rho}(x) \frac{\partial \phi^{a}}{\partial x^{\rho}} \frac{\partial \phi^{b}}{\partial x^{\nu}} \eta_{a b}(\phi)$,

is obtained.

One interesting property with this massive gravity nonlinear sigma model is that its strong coupling scale is given by $\Lambda_{2}$. This is higher than $\Lambda_{3} \equiv\left(\mathrm{m}^{2} / \kappa_{g}\right)^{1 / 3}$, considered as the highest possible strong coupling scale in ghost-free massive gravity, coming from the analysis around the trivial vacuum $g_{\mu \nu}=\eta_{\mu \nu}, \phi^{a}=x^{a}$. Another interesting property is that the vector and scalar modes of the graviton encoded in $\phi^{a}$ decouple with matter fields even in the linear regime, which does not give the van Dam-Veltman-Zakharov discontinuity and the Vainshtein mechanism is implemented automatically.

This suggests that as long as the Vainshtein screening works, even if we start with a more general setup described by Eq. (2.1), that is, not limiting $g_{\mu \nu}$ and $f_{a b}$ to flat, not neglecting the $f$-matter fields, we can expect that the massive gravity nonlinear sigma model is obtained as an effective theory inside the Vainshtein radius. Actually, if the Vainshtein mechanism is working, the metrics can be expressed by

$$
\begin{aligned}
& g_{\mu \nu}=g_{\mu \nu}^{\mathrm{GR}}+\kappa_{g} \delta g_{\mu \nu}, \\
& f_{a b}=f_{a b}^{\mathrm{GR}}+\kappa_{f} \delta f_{a b},
\end{aligned}
$$

where $\delta g_{\mu \nu}$ and $\delta f_{a b}$ should be treated as perturbations. The metrics $g_{\mu \nu}^{\mathrm{GR}}$ and $f_{a b}^{\mathrm{GR}}$ are assumed to be solutions in GR with the matter actions $S_{g}^{[\mathrm{m}]}$ and $S_{f}^{[\mathrm{m}]}$, respectively, in order to remove the tadpole terms for $\delta g_{\mu \nu}$ and $\delta f_{a b}$ in the $\Lambda_{2}$ decoupling limit. ${ }^{2}$ Here, $g_{\mu \nu}^{\mathrm{GR}}(x)$ and $f_{a b}^{\mathrm{GR}}(\phi)$ are determined as functions of $x^{\mu}$ and $\phi^{a}$, respectively, from which we can regard that $g_{\mu \nu}^{\mathrm{GR}}$ and $f_{a b}^{\mathrm{GR}}$ act as external forces. Then with the undetermined variables $\phi^{a}, \delta g_{\mu \nu}, \delta f_{a b}$, the action can be expanded as

\footnotetext{
${ }^{2}$ In the case of massive gravity, the $f$-metric can be a generic metric rather than a GR solution since the Einstein-Hilbert action for $f_{\mu \nu}$ is absent.
} 


$$
\begin{aligned}
S= & S_{\mathrm{GR}}(\delta g)+S_{\mathrm{GR}}(\delta f) \\
& +S_{\mathrm{MG}-\mathrm{NLS}}\left(\phi^{a} ; g^{\mathrm{GR}}, f^{\mathrm{GR}}\right)+\Lambda_{2}^{4} \mathcal{O}\left(\kappa_{g} \delta g, \kappa_{f} \delta f\right),
\end{aligned}
$$

where $S_{\mathrm{GR}}$ are the perturbed actions for the metric perturbations, which are the same as those in GR. $S_{\mathrm{MG}-\mathrm{NLS}}$ is the action of the massive gravity nonlinear sigma model given by

$S_{\mathrm{MG}-\mathrm{NLS}}=-\Lambda_{2}^{4} \int d^{4} x \sqrt{-g_{\mathrm{GR}}} \sum_{n=2}^{4} c_{n} \mathcal{U}_{n}\left(\gamma_{\mathrm{NLS}}\right)$,

which generalizes Eq. (2.13) with the following replacements:

$$
\kappa_{g} \rightarrow \kappa, \quad \eta_{\mu \nu} \rightarrow g_{\mu \nu}^{\mathrm{GR}}, \quad \eta_{a b} \rightarrow f_{a b}^{\mathrm{GR}} .
$$

One may worry that the tadpole terms of the metric perturbations give the backreaction from the Stuieckelberg fields to the spacetimes. Since the background spacetimes are given by the solutions in GR, they appear only through the interaction terms between the Stuieckelberg fields and the metric perturbations of order $\Lambda_{2}^{4} \mathcal{O}\left(\kappa_{g} \delta g, \kappa_{f} \delta f\right)$. However, by taking the $\Lambda_{2}$ decoupling limit given by Eq. (2.7), the contributions from the tadpole terms are negligible and then the Stüeckelberg fields and the metric perturbations are decoupled. Then, in this limit the Stüeckelberg fields are simply determined by the massive gravity nonlinear sigma model (2.13) and the spacetimes are completely the same as those in GR. Therefore the massive gravity nonlinear sigma model with curved metrics is the effective action of the Stuieckelberg fields as long as the Vainshtein mechanism works and we have the same solutions as in GR.

Indeed, the Vainshtein screening solutions can be obtained by this effective action with curved metrics. The Vainshtein mechanism for the static and spherically symmetric spacetime is found with the interior and the exterior Schwarzschild metrics [47] (see also [42,45]) and the cosmological Vainshtein mechanism is found with the Friedmann-Lemaître-Robertson-Walker metric [46]. Although we denoted the procedure of the $\Lambda_{2}$ decoupling limit just as the massless limit in these papers, the limits used in [46,47] are equivalent to the $\Lambda_{2}$ decoupling limit shown above.

For simplicity, we do not introduce the $f$-matter fields in this paper; thus we can assume that $f_{a b}^{\mathrm{GR}}$ is the Minkowski spacetime. In this case, the Stüeckelberg field can be split as $\phi^{a}=\delta_{\mu}^{a}\left(x^{\mu}+\pi^{\mu}\right)$; then $f_{\mu \nu}$ is expressed by

$$
f_{\mu \nu}=\eta_{\mu \nu}+2 \partial_{(\mu} \pi_{\nu)}+\partial_{\mu} \pi_{\alpha} \partial_{\nu} \pi^{\alpha},
$$

where $\pi_{\mu}$ is a vector field on the Minkowski spacetime. We shall discuss the action (2.13) as the effective action inside the Vainshtein radius.

\section{SCALAR MODE INSTABILITY: GENERAL BACKGROUND}

In this section, we show that the scalar graviton generally suffers from a ghost and/or a gradient instability when there is no vector graviton excitation in the massive gravity nonlinear sigma model. To see the existence of the instability, we assume a weak gravitational field and ignore the vector graviton excitation:

$$
\begin{gathered}
g_{\mu \nu}^{\mathrm{GR}}=\eta_{\mu \nu}+h_{\mu \nu}^{\mathrm{GR}}, \\
\pi_{\mu}=\partial_{\mu} \pi .
\end{gathered}
$$

In a similar way to the $\Lambda_{3}$ decoupling limit [20], the action can be expanded as

$$
\begin{aligned}
\mathcal{L}_{\mathrm{MG}-\mathrm{NLS}}= & -\frac{\Lambda_{2}^{4}}{2} h^{\mathrm{GR} \mu \nu}\left(X_{\mu \nu}^{(1)}+\beta_{2} X_{\mu \nu}^{(2)}+\beta_{3} X_{\mu \nu}^{(3)}\right) \\
& +\mathcal{O}\left(h_{\mathrm{GR}}^{2}\right),
\end{aligned}
$$

where

$$
\beta_{2}=c_{3}-1, \quad \beta_{3}=-\left(c_{3}+c_{4}\right),
$$

and

$$
\begin{aligned}
& X_{\mu \nu}^{(1)}=-\frac{1}{2} \epsilon_{\mu}^{\alpha \beta \gamma} \epsilon_{\nu \alpha^{\prime} \beta \gamma} \Pi_{\alpha}^{\alpha^{\prime}}=[\Pi] \eta_{\mu \nu}-\Pi_{\mu \nu}, \\
& X_{\mu \nu}^{(2)}=-\frac{1}{2} \epsilon_{\mu}^{\alpha \beta \gamma} \epsilon_{\nu \alpha^{\prime} \beta^{\prime} \gamma} \Pi_{\alpha}^{\alpha^{\prime}} \Pi_{\beta^{\prime}}^{\beta} \\
& =\frac{1}{2} \eta_{\mu \nu}\left([\Pi]^{2}-\left[\Pi^{2}\right]\right)+\Pi_{\mu \nu}^{2}-[\Pi] \Pi_{\mu \nu}, \\
& X_{\mu \nu}^{(3)}=-\frac{1}{3} \epsilon_{\mu}^{\alpha \beta \gamma} \epsilon_{\nu \alpha^{\prime} \beta^{\prime} \gamma^{\prime}} \Pi_{\alpha}^{\alpha^{\prime}} \Pi_{\beta}^{\beta^{\prime}} \Pi_{\gamma}^{\gamma^{\prime}} \\
& =\frac{1}{6}\left([\Pi]^{3}-3[\Pi]\left[\Pi^{2}\right]+2\left[\Pi^{3}\right]\right) \eta_{\mu \nu} \\
& -\Pi_{\mu \nu}^{3}+[\Pi] \Pi_{\mu \nu}^{2}-\frac{1}{2}\left([\Pi]^{2}-\left[\Pi^{2}\right]\right) .
\end{aligned}
$$

Here, we have introduced the notation $\Pi_{\mu \nu}=\partial_{\mu} \partial_{\nu} \pi$, $\Pi^{n \mu}{ }_{\nu}=\Pi^{\mu}{ }_{\alpha_{2}} \Pi^{\alpha_{2}}{ }_{\alpha_{3}} \cdots \Pi^{\alpha_{n}}{ }_{\nu}$, and $\left[\Pi^{n}\right]=\Pi^{n \mu}{ }_{\mu}$. Note that, differently from the case of the $\Lambda_{3}$ decoupling limit, $h_{\mu \nu}^{\mathrm{GR}}$ has been already fixed and it acts as an external force for the field $\pi$.

The field $\pi$ can be split into the background configuration $\bar{\pi}$ and the perturbation $\delta \pi$ as

$$
\pi=\bar{\pi}+\delta \pi,
$$

with $\delta \pi \ll \bar{\pi}$. $\bar{\pi}$ is determined by the equation of motion 


$$
\begin{aligned}
\epsilon^{\alpha \beta \gamma \delta} \epsilon_{\mu \nu \rho \sigma} R^{(1) \mu \nu}{ }_{\alpha \beta}\left(\delta^{\rho}{ }_{\gamma} \delta^{\sigma}{ }_{\delta}+2 \beta_{2} \bar{\Pi}_{\gamma}{ }_{\gamma} \delta^{\sigma}{ }_{\delta}+\beta_{3} \bar{\Pi}^{\rho}{ }_{\gamma} \bar{\Pi}^{\sigma}{ }_{\delta}\right) \\
=\epsilon^{\alpha \beta \gamma \delta} \epsilon_{\mu \nu \rho \sigma} \partial^{\mu}\left[\left(\partial_{\beta} h^{\mathrm{GR} \nu}{ }_{\alpha}-\partial_{\alpha} h^{\mathrm{GR} \nu}{ }_{\beta}\right)\right. \\
\left.\times\left(\delta^{\rho}{ }_{\gamma} \delta^{\sigma}{ }_{\delta}+2 \beta_{2} \bar{\Pi}^{\rho}{ }_{\gamma} \delta^{\sigma}{ }_{\delta}+\beta_{3} \bar{\Pi}^{\rho}{ }_{\gamma} \bar{\Pi}^{\sigma}{ }_{\delta}\right)\right]=0,
\end{aligned}
$$

where

$$
R_{\mu \nu \alpha \beta}^{(1)}=\partial_{\mu} \partial_{[\beta} h_{\alpha] \nu}^{\mathrm{GR}}+\partial_{\nu} \partial_{[\alpha} h_{\beta] \mu}^{\mathrm{GR}},
$$

is the linearized Riemann curvature and $\bar{\Pi}_{\mu \nu}=\partial_{\mu} \partial_{\nu} \bar{\pi}$. Then the quadratic order action for the perturbation $\delta \pi$ is given by

$$
\mathcal{L}_{2}=-\frac{1}{2} Z^{\mu \nu} \partial_{\mu} \delta \pi \partial_{\nu} \delta \pi+\mathcal{O}\left(h_{\mathrm{GR}}^{2}\right),
$$

where

$Z^{\mu \nu}=-\frac{\Lambda_{2}^{4}}{4} \epsilon^{\mu \alpha \beta \gamma} \epsilon_{\alpha^{\prime} \beta^{\prime} \gamma^{\prime}} R^{(1) \alpha^{\prime} \beta^{\prime}}{ }_{\alpha \beta}\left(\beta_{2} \delta^{\gamma^{\prime}}{ }_{\gamma}+\beta_{3} \bar{\Pi}^{\gamma^{\prime}}{ }_{\gamma}\right)$.

We note that

$$
\begin{aligned}
Z^{\mu \nu}= & \beta_{2} \times(\text { Ricci curvatures }) \\
& +\beta_{3} \times \bar{\Pi} \times(\text { Riemann and Ricci curvatures }) .
\end{aligned}
$$

Thus $Z^{\mu \nu}$ is identically zero for a Ricci flat spacetime when $\beta_{3}=0$. For this case we should take into account next order contributions of $h_{\mathrm{GR}}$. In this paper, however, we restrict our analysis to the case of $\beta_{3} \neq 0$ and assume that $Z^{\mu \nu}$ is not zero.

The no-ghost and no-gradient instability condition is given by the signs of eigenvalues of $Z^{\mu \nu}$; these are $[-,+,+,+]$, which is equivalent to all eigenvalues of $Z^{\mu}{ }_{\nu}$ being positive. Hence we obtain

$$
Z_{\mu}^{\mu}>0,
$$

as a necessary condition of no instabilities. However, we obtain

$$
Z^{\mu}{ }_{\mu} \propto \text { Ricci curvatures. }
$$

Since the sum of the eigenvalues is zero for any Ricci flat spacetime, there is at least one negative eigenvalue of $Z^{\mu}{ }_{\nu}$, which leads to a ghost instability or a gradient instability. As a result a ghost and/or a gradient instability appears for any Ricci flat background.

For instance, the static and spherically symmetric solution is given by

$h_{t t}^{\mathrm{GR}}=\frac{2 G M}{r}, \quad h_{r r}^{\mathrm{GR}}=\frac{2 G M}{r}, \quad$ others $=0$, $\partial_{\mu} \bar{\pi}=(0, r \mu(r), 0,0), \quad \mu= \pm \frac{1}{\sqrt{\beta_{3}}}+\mathcal{O}(G M / r)$.

Then the matrix $Z^{\mu \nu}$ is given by

$Z^{\mu \nu}=\frac{G M \sqrt{\beta_{3}}}{r^{3}} \times \operatorname{diag}\left[0, \mp 2, \pm \frac{1}{r^{2}}, \pm \frac{1}{r^{2} \sin ^{2} \theta}\right]$.

Hence the gradient instability appears from either the radial or the angular derivatives. Note that, since the $(t t)$ component of $Z^{\mu \nu}$ is zero at leading order of $G M / r$, it seems that the scalar graviton is infinitely strongly coupled. However, the kinetic term indeed appears at the next order of $G M / r$ and our effective action does not lose any degrees of freedom as we will see in the next section.

Since the bigravity theory contains degrees of freedom of the vector graviton as well as one of the scalar graviton and these are coupled to each other in a general background, one cannot directly conclude that the Vainshtein screening solution is unstable in a Ricci flat background spacetime. Therefore we shall discuss general perturbations including vector gravitons around the static and spherically symmetric background in the next section. Regardless of this, our result suggests that the Vainshtein screening solutions cannot be supported only by the scalar graviton and that the excitation of the vector graviton has to be taken into account.

Note that our result can also be straightforwardly applied to the Horndeski theories, which was discussed in [17]. Based on the effective action for the Vainshtein mechanism, the paper [17] showed the static and spherically symmetric solution with the Vainshtein screening is unstable as long as the Horndeski theory includes the so-called $\mathcal{L}_{5}$ term. Actually, for the case of Horndeski theory, although other terms appear in the effective action, they are shown to be subdominant if we assume that the Vainshtein screening is working, which gives the same action as (3.11) (see Appendix).

\section{INSTABILITY OF STATIC AND SPHERICALLY SYMMETRIC SOLUTION}

In the previous section, we have shown that the Vainshtein screening solutions cannot be supported only by the excitation of the scalar graviton. However, since the massive gravity nonlinear sigma model contains the degrees of freedom of the vector graviton as well, one cannot still conclude that the solution is indeed unstable. In this section, thus, we study the general perturbations around the static and spherically symmetric solutions with Vainshtein screening given by $[42,45,47]$, in which there exists only the scalar graviton in the background solution. For simplicity, we only focus on the space region outside the source $\left(r>R_{\star}>2 G M\right)$; thus the $g$-spacetime is given 
by the Schwarzschild spacetime. We choose the spherical coordinate

$$
\begin{aligned}
g_{\mu \nu}^{\mathrm{GR}} & =\operatorname{diag}\left[-F_{g}^{2}, F_{g}^{-2}, r^{2}, r^{2} \sin ^{2} \theta\right], \\
\eta_{\mu \nu} & =\operatorname{diag}\left[-1,1, r^{2}, r^{2} \sin ^{2} \theta\right],
\end{aligned}
$$

with $F_{g}^{2}=1-\frac{2 G M}{r}$, where $M$ and $R_{\star}$ is the gravitational mass and radius of the star, respectively.
First we give the static and spherically symmetric solution. The solution can be found by assuming the Stüeckelberg field as

$$
\pi_{\mu}=\bar{\pi}_{\mu}=(0, r \mu(r), 0,0) .
$$

The basic equation can be derived by varying the action with respect to $\mu$. Then the solution is given by

$\mu=\frac{-\left(1-F_{g}\right)\left(\beta_{2}-2 \beta_{3}\right)+\epsilon \sqrt{\left(\beta_{2}-2 \beta_{3}\right)^{2}\left(1-F_{g}\right)^{2}+\beta_{3}\left(1+F_{g}\right)\left(4 \beta_{2}-1+\left(3-4 \beta_{2}\right) F_{g}\right)}}{\beta_{3}\left(1+F_{g}\right)}=\frac{\epsilon}{\sqrt{\beta_{3}}}+\mathcal{O}(G M / r)$,

where $\epsilon= \pm 1$. Note that although $\beta_{2}$ and $\beta_{3}$ are originally free parameters, it was shown that this solution exists only for $\beta_{3}>1$ with some other constraints on $\beta_{2}$ and $\beta_{3}$ in bigravity $[45,47]$. Note also that although the solution (4.4) with the metrics (4.1) and (4.2) is obtained by the effective action (2.13), this solution is indeed the approximate solution deep inside the Vainshtein radius in the bigravity theory [47]. Here the minus branch $(\epsilon=-1)$ is the asymptotically flat branch. On the other hand the plus branch $(\epsilon=1)$ is not regular in general; however, the plus branch can describe a regular asymptotically AdS solution when we introduce a negative cosmological constant.

We shall study the stability of this solution. Since the background spacetime is spherically symmetric, perturbations can be decomposed into odd parity perturbations and even parity perturbations, that is,

$$
\pi_{\mu}=\bar{\pi}_{\mu}+\delta \pi_{\mu}^{\mathrm{odd}}+\delta \pi_{\mu}^{\mathrm{even}}
$$

These perturbations are decoupled at the linear order equation of motion (or equivalently at the quadratic order Lagrangian). Hence we separately discuss the odd and even parity perturbations, in order.

\section{A. Odd parity perturbations}

First we discuss the odd parity perturbations. Because of the spherical symmetry of the background solution, we can separate the variables and then the $x^{i}=(\theta, \varphi)$ dependence can be expanded in terms of the vector spherical harmonics $Y_{i}$, which is defined by

$$
\begin{gathered}
{\left[D^{2}+\ell(\ell+1)-1\right] Y_{i}=0,(\ell=1,2, \cdots),} \\
D^{i} Y_{i}=0 .
\end{gathered}
$$

Here, $D_{i}$ is the covariant derivative on the 2-sphere and $D^{2}=D_{i} D^{i}$. The explicit form of $Y_{i}$ is given by

$$
Y_{i}=\epsilon_{i j} D^{j} Y
$$

where $\epsilon_{i j}$ is the Levi-Cività tensor and $Y$ is the spherical harmonics satisfying

$$
\left[D^{2}+\ell(\ell+1)\right] Y=0, \quad(\ell=0,1,2, \cdots) .
$$

By using the vector harmonics, the perturbation of the Stiueckelberg field is expressed by

$$
\delta \pi_{\mu}^{\text {odd }}=\left(0,0, r \chi_{\Omega} Y_{i}\right),
$$

where $\chi_{\Omega}$ is a function of $(t, r)$.

The quadratic order action is given by

$$
\begin{aligned}
S^{\text {odd }}= & \int r^{2} d t d r d \Omega \frac{\Lambda_{2}^{4}}{4}\left(\sqrt{\beta_{3}}+\epsilon \beta_{2}\right) \\
& \times\left[\frac{4}{2 \sqrt{\beta_{3}}+\epsilon} \dot{\chi}_{\Omega}^{2}-\frac{1}{\sqrt{\beta_{3}}+\epsilon}\left(\chi_{\Omega}^{\prime 2}+\frac{\ell(\ell+1)}{r^{2}} \chi_{\Omega}^{2}\right)\right] Y_{i} Y^{i} \\
& +\mathcal{O}(G M / r),
\end{aligned}
$$

where a dot and a prime denote the time derivative and the radial derivative, respectively. Since each eigenmode of the harmonics does not couple with the other eigenmodes, we drop the summation sign. The stability condition (no-ghost and no-gradient instability) is given by

$$
\sqrt{\beta_{3}}+\beta_{2}>0
$$

for the plus branch, while for the minus branch the condition is

$$
\sqrt{\beta_{3}}-\beta_{2}>0 \text {. }
$$

\section{B. Even parity perturbations}

Next we consider the even parity perturbations. By using the spherical harmonics, the perturbation of the Stüeckelberg field is expressed by 


$$
\delta \pi_{\mu}^{\mathrm{even}}=\left(\xi_{t} Y, \xi_{r} Y, r \xi_{\Omega} D_{i} Y\right),
$$

where $\xi_{t}, \xi_{r}$, and $\xi_{\Omega}$ are functions of $(t, r)$. Note that for the $\ell=0$ mode, the variable $\xi_{\Omega}$ is undefined because $D_{i} Y=0$. Hence we should discuss the $\ell=0$ mode and $\ell \geq 1$ modes, separately.

\section{Radial perturbation $(\boldsymbol{\ell}=0)$}

For $\ell=0$, the spherical harmonics is simply given by $\left.Y\right|_{\ell=0}=1 / \sqrt{4 \pi}$. The quadratic order action can be schematically expressed by

$$
S^{\ell=0}=S^{\ell=0}\left(\dot{\xi}_{r}, \xi_{t}^{\prime}, \xi_{r}^{\prime}, \xi_{t}, \xi_{r}\right),
$$

from which $\xi_{t}$ is nondynamical and it can be integrated out. The variation with respect to $\xi_{t}$ yields a constraint equation

$$
\begin{aligned}
& \partial_{t} {\left[2 r F_{g}^{-1}\left(F_{g}-1\right)\left(\beta_{2}+\beta_{3} \mu\right) \xi_{r}\right]-\partial_{r} } \\
& \quad \times\left[\frac{r^{2} F_{g}\left(1+2 \beta_{2} \mu+\beta_{3} \mu^{2}\right)\left(F_{g}^{2} \xi_{t}^{\prime}-\dot{\xi}_{r}\right)}{1+F_{g}^{2}(r+r \mu)^{\prime}}\right]=0,
\end{aligned}
$$

where the solution is given by

$$
\begin{aligned}
\xi_{t}^{\prime}= & -2 G M\left(\beta_{2}+\epsilon \sqrt{\beta_{3}}\right)\left[\frac{\dot{\Xi}^{\prime}}{2 r F_{g}\left(F_{g}-1\right)\left(\beta_{2}+\beta_{3} \mu\right)}\right. \\
& \left.+\frac{1+F_{g}^{2}(r+r \mu)^{\prime}}{r^{2} F_{g}^{3}\left(1+2 \beta_{2} \mu+\beta_{3} \mu^{2}\right)} \dot{\Xi}\right], \\
\xi_{r}= & -2 G M\left(\beta_{2}+\epsilon \sqrt{\beta_{3}}\right) \frac{F_{g}}{2 r\left(F_{g}-1\right)\left(\beta_{2}+\beta_{3} \mu\right)} \Xi^{\prime},
\end{aligned}
$$

with some function $\Xi(t, r)$. Here the factor is introduced so that $\xi_{t}$ and $\xi_{r}$ can be expressed by

$$
\begin{aligned}
& \xi_{t}=\dot{\Xi}+\mathcal{O}(G M / r), \\
& \xi_{r}=\Xi^{\prime}+\mathcal{O}(G M / r),
\end{aligned}
$$

at the leading order of $G M / r$. Then the quadratic action is expressed by

$$
S^{\ell=0}=\Lambda_{2}^{4} \int r^{2} d t d r\left[K_{t} \dot{\Xi}^{2}-K_{r} \Xi^{\prime 2}\right],
$$

where

$K_{t}=-\epsilon\left(\frac{G M}{r}\right)^{2} \frac{3 \sqrt{\beta_{3}}\left(\beta_{2}+\epsilon \sqrt{\beta_{3}}\right)}{r^{2}}+\mathcal{O}\left(\left(\frac{G M}{r}\right)^{3}\right)$

$K_{r}=-\epsilon \frac{G M}{r} \frac{\sqrt{\beta_{3}}}{r^{2}}+\mathcal{O}\left(\left(\frac{G M}{r}\right)^{2}\right)$.
Note that, while the gradient term appears at the first order of $G M / r$ (i.e., the first order of the metric perturbation around the Minkowski spacetime), the kinetic term appears at the second order of $G M / r$. Hence the scalar graviton is not infinitely strongly coupled, although the propagation speed is superluminal.

From the second order action, we can see that the plus branch suffers from the gradient instability. Even for the minus branch, the stability condition is given by

$$
\beta_{2}-\sqrt{\beta_{3}}>0,
$$

which has a sign opposite to the stability condition of the odd parity perturbations. As a result we conclude that the static spherically symmetric solution is unstable for any parameters of $\beta_{2}$ and $\beta_{3}$.

\section{General modes $(\ell \geq 1)$}

Although we have shown the instability of the background solution, we discuss general modes of the even parity perturbations for completeness. The quadratic action can be expressed by

$$
S^{\text {even }}=\int Y^{2} d \Omega \int d t d r \mathcal{L}^{\text {even }}\left(\dot{\xi}_{r}, \dot{\xi}_{\Omega}, \xi_{A}^{\prime}, \xi_{A}\right),
$$

where $A=(t, r, \Omega)$; thus $\xi_{t}$ is a nondynamical variable, same as the case of the $\ell=0$ mode. However, contrary to the case of the $\ell=0$ mode, the constraint equation, which is derived by the variation with respect to $\xi_{t}$, is not easily solved. We notice, however, that the constraint equation has a particular solution

$$
\delta \pi_{\mu}^{\mathrm{even}}=\partial_{\mu}(\Xi(t, r) Y(\theta, \varphi))+\mathcal{O}(G M / r),
$$

in which there is no degree of freedom of the vector graviton. Since the stability of the case of the purely scalar graviton has already been discussed in the previous section, we shall not discuss this case furthermore here.

To discuss the stability of the general perturbations, we use the Hamiltonian formulation and calculate the on-shell Hamiltonian. The canonical momenta are defined by

$$
\pi_{A}=\frac{\delta \mathcal{L}^{\text {even }}}{\delta \dot{\xi}_{A}} .
$$

Since the Lagrangian does not contain $\dot{\xi}_{t}$, there is a primary constraint

$$
\Phi^{1}:=\pi_{t} \approx 0,
$$

where the symbol " $\approx$ " is the weak equality which holds on shell. $\dot{\xi}_{r}$ and $\dot{\xi}_{\Omega}$ can be expressed in terms of canonical variables. Then the total Hamiltonian is given by 


$$
\begin{aligned}
\mathcal{H}_{T}^{\text {even }} & =\pi_{r} \dot{\xi}_{r}+\pi_{\Omega} \dot{\xi}_{\Omega}-\mathcal{L}^{\text {even }}+\lambda \pi_{t} \\
& =\mathcal{H}^{\text {even }}\left[\pi_{r}, \pi_{\Omega}, \xi_{A}\right]+\lambda \pi_{t},
\end{aligned}
$$

where $\lambda$ is the Lagrangian multiplier. The preservation of the primary constraint yields

$$
\Phi^{2}:=\left\{\Phi^{1}, H_{T}^{\text {even }}\right\} \approx 0,
$$

where

$$
H_{T}^{\text {even }}=\int d r \mathcal{H}_{T}^{\text {even }}
$$

Note that since $\Phi^{2}$ contains only $\xi_{t}, \pi_{r}, \pi_{r}^{\prime}$, and $\pi_{\Omega}$, the secondary constraint $\Phi^{2} \approx 0$ is the constraint equation on the canonical variables, from which we can easily express $\xi_{t}$ in terms of $\pi_{r}, \pi_{r}^{\prime}$, and $\pi_{\Omega}$. This system has only these two constraints. Indeed, the condition $\left\{\Phi^{2}, H_{T}^{\text {even }}\right\} \approx 0$ contains the Lagrangian multiplier $\lambda$ and it does not generate a constraint equation, but an equation to determine the Lagrangian multiplier. As a result, we have two constraint equations on the canonical variables, which are second class. Hence the degree of freedom of this system in the phase space is

$$
\text { d.o.f. }=6-2=2 \times 2 \text {, }
$$

which indicates that the even parity perturbations contain one scalar graviton and one vector graviton.

Substituting the solutions of the constraint equations into the Hamiltonian, the on-shell Hamiltonian is given by

$$
\begin{aligned}
H_{\text {on-shell }}^{\text {even }} & =\int d r \mathcal{H}_{\text {on-shell }}^{\text {even }}\left(\pi_{r}, \pi_{r}^{\prime}, \pi_{\Omega}, \xi_{r}, \xi_{\Omega}, \xi_{\Omega}^{\prime}\right), \\
& =\int d r \Lambda_{2}^{4}\left[\frac{K_{1}}{r^{2}}\left(\pi_{r}+A_{1} \pi_{\Omega}\right)^{2}\right. \\
& +\frac{K_{2}}{r^{2}}\left(r \pi_{r}^{\prime}+A_{2} \pi_{\Omega}\right)^{2}+\frac{K_{3}}{r^{2}} \pi_{\Omega}^{2} \\
& \left.+K_{4}\left(\xi_{\Omega}+A_{4} \xi_{r}\right)^{2}+K_{5}\left(r \xi_{\Omega}^{\prime}+A_{5} \xi_{r}\right)^{2}+K_{6} \xi_{r}^{2}\right],
\end{aligned}
$$

where the dimensionless coefficients are expanded as

$$
\begin{aligned}
& K_{1}=\epsilon \frac{\mathcal{B}_{1}}{48 \beta_{3}^{3 / 2}\left(\beta_{2}+\epsilon \sqrt{\beta_{3}}\right)}+\mathcal{O}\left(\frac{G M}{r}\right), \\
& K_{2}=-\epsilon\left(\frac{G M}{r}\right)^{-2} \frac{1}{12 \sqrt{\beta_{3}}\left(\beta_{2}+\epsilon \sqrt{\beta_{3}}\right)}+\mathcal{O}\left(\left(\frac{G M}{r}\right)^{-1}\right), \\
& K_{3}=-\epsilon\left(\frac{G M}{r}\right)^{-2} \frac{\sqrt{\beta_{3}}\left(1-2 \epsilon \sqrt{\beta_{3}}\right)^{2}}{3 \mathcal{B}_{1}\left(\beta_{2}+\epsilon \sqrt{\beta_{3}}\right)}+\mathcal{O}\left(\left(\frac{G M}{r}\right)^{-1}\right),
\end{aligned}
$$

$$
K_{4}=-\epsilon \ell(\ell+1) \frac{G M}{r} \frac{\mathcal{B}_{2}}{16 \sqrt{\beta_{3}}\left(\epsilon+\sqrt{\beta_{3}}\right)^{2}}+\mathcal{O}\left(\left(\frac{G M}{r}\right)^{2}\right) \text {, }
$$

$$
K_{5}=\epsilon \ell(\ell+1) \frac{\beta_{2}+\epsilon \sqrt{\beta_{3}}}{4\left(\epsilon+\sqrt{\beta_{3}}\right)}+\mathcal{O}\left(\frac{G M}{r}\right)
$$

$$
K_{6}=\epsilon \ell(\ell+1)\left(\frac{G M}{r}\right)^{-1} \sqrt{\beta_{3}} \mathcal{B}_{2}^{-1}\left(\beta_{2}+\epsilon \sqrt{\beta_{3}}\right)^{2}+\mathcal{O}(1),
$$

and

$$
\begin{aligned}
& A_{1}=\left(\frac{G M}{r}\right)^{-1} 4 \beta_{3} \mathcal{B}_{1}^{-1}\left(1-2 \epsilon \sqrt{\beta_{3}}\right)+\mathcal{O}(1), \\
& A_{2}=-1+\mathcal{O}\left(\frac{G M}{r}\right) \\
& A_{4}=\left(\frac{G M}{r}\right)^{-1} 4 \sqrt{\beta_{g}} \mathcal{B}_{2}^{-1}\left(\epsilon+\sqrt{\beta_{3}}\right)\left(\beta_{2}+\epsilon \sqrt{\beta_{3}}\right)+\mathcal{O}(1),
\end{aligned}
$$

$A_{5}=-1+\mathcal{O}\left(\frac{G M}{r}\right)$

with

$$
\begin{aligned}
\mathcal{B}_{1}:= & \beta_{2}+8 \beta_{3}-4 \beta_{2} \beta_{3}+\epsilon \sqrt{\beta_{3}}\left(4 \beta_{3}-3\right), \\
\mathcal{B}_{2}:= & \beta_{2}^{2}\left(1-4 \beta_{3}\right)+\beta_{3}\left(5+4 \beta_{3}\right)-4 \beta_{2} \beta_{4} \\
& +2 \epsilon \sqrt{\beta_{3}}\left(6 \beta_{3}-\beta_{2}\left(1+4 \beta_{3}\right)\right) .
\end{aligned}
$$

One can find $K_{1} K_{3}<0$ and $K_{4} K_{6}<0$ for any parameters $\left(\beta_{2}, \beta_{3}\right)$; thus the Hamiltonian is unbounded from below, which means that the perturbations suffer from the instability.

\section{SUMMARY AND DISCUSSION}

In this paper, we showed that the massive gravity nonlinear sigma model gives an effective theory of the vector and scalar gravitons inside the Vainshtein radius for general massive gravity/bigravity. We obtained the effective action by taking the $\Lambda_{2}$ decoupling limit around a curved spacetime and it can be used as long as we have the Vainshtein screening solutions. Making use of the massive gravity nonlinear sigma model as the effective action inside the Vainshtein radius, we studied the stability of the Vainshtein screening solutions in massive gravity/bigravity. 
First we derived a general consequence that in any Ricci flat background spacetime, the scalar graviton generally suffers from a ghost and/or a gradient instability as long as the vector graviton is not excited. Since the spacetime is given by a solution in GR, the Ricci flat region is realized by the vacuum region of the spacetime; thus the instability is found outside the source. However since the massive gravity/bigravity contains the vector graviton and the perturbations of scalar and vector gravitons are coupled, one cannot directly conclude that the Ricci flat Vainshtein screening background spacetime is indeed unstable.

Hence we studied perturbations around the static and spherically symmetric solution obtained in Ref. [47] next. We clarified the stability condition for both odd parity perturbations and even parity perturbations, which depends on $\beta_{2}$ and $\beta_{3}$, model parameters in the massive gravity nonlinear sigma model, and $\epsilon$, a parameter depending on the asymptotic behavior of the background solution. As a result, for any parameters $\left(\beta_{2}, \beta_{3}, \epsilon\right)$, we found that the perturbations suffer from some of the instabilities and confirmed that the Vainshtein screening background solution is unstable.

We have shown the (local) instability of the spherically symmetric solution in the space region outside the star. In addition, the instability of a black hole solution was shown in [54,55] (see also [56-59]). Note that our background solution completely differs from the background solution discussed in [54,55]. For the black hole solution, both metrics are given by the same Schwarzschild metric (or Kerr metric) in which the Stüeckelberg fields are not excited, i.e., $\phi^{a}=x^{a}$. One may expect that there exists a stable hairy black hole supported by the hair of the Stuieckelberg fields. However, our result suggests that a scalar graviton hair is not helpful for supporting astrophysical objects. In particular, existence of a spherically symmetric hairy black hole is unlikely as numerically shown in [60].

The instability implies the difficulty of constructing viable astrophysical objects in the context of massive gravity/bigravity. The universality of the instability suggests that the Vainshtein screening could not be realized only by the scalar graviton. To obtain a stable solution with the Vainshtein screening, the vector graviton has to be nonlinearly excited in a vacuum region of the spacetime. Therefore it is also important to study the property of the vector graviton in more general spacetimes for the Vainshtein mechanism in massive gravity/bigravity.

\section{ACKNOWLEDGMENTS}

K. A. would like to thank Shinji Mukohyama for useful discussions and comments. His work was supported in part by Grants-in-Aid from the Scientific Research Fund of the Japan Society for the Promotion of Science (JSPS), Grant No. 15J05540. This work was supported in part by the JSPS Grant-in-Aid for Scientific Research No. 16K17709 (S. M.).

\section{APPENDIX: SCALAR MODE INSTABILITY IN THE HORNDESKI THEORY}

In this appendix we briefly discuss the stability of the Vainshtein mechanism in the Horndeski theory, the most general scalar-tensor theory in the sense that its equation of motion contains up to the second derivative of the fields. The effective theory for the Vainshtein mechanism around the Minkowski spacetime [17] is given by the Lagrangian

$$
\begin{aligned}
\mathcal{L}= & -\frac{1}{4} h^{\mu \nu} \mathcal{E}_{\mu \nu, \alpha \beta} h^{\alpha \beta}+\sum_{n=2}^{5} \frac{\tilde{\alpha}_{n}}{\Lambda_{3}^{3(n-2)}} \mathcal{L}_{n}^{\mathrm{gal}} \\
& -\frac{1}{2} \sum_{n=1}^{3} \frac{\tilde{\beta}_{n}}{\Lambda_{3}^{3(n-1)}} h^{\mu \nu} X_{\mu \nu}^{(n)}+\frac{1}{2 M_{\mathrm{pl}}} h_{\mu \nu} T^{\mu \nu},
\end{aligned}
$$

where

$$
\begin{aligned}
\mathcal{L}_{2}^{\text {gal }} & =-\frac{1}{2}(\partial \pi)^{2}, \\
\mathcal{L}_{3}^{\text {gal }} & =-\frac{1}{2}(\partial \pi)^{2}[\Pi], \\
\mathcal{L}_{4}^{\text {gal }} & =-\frac{1}{2}(\partial \pi)^{2}\left([\Pi]^{2}-\left[\Pi^{2}\right]\right), \\
\mathcal{L}_{5}^{\text {gal }} & =-\frac{1}{12}(\partial \pi)^{2}\left([\Pi]^{3}-3[\Pi]\left[\Pi^{2}\right]+2\left[\Pi^{3}\right]\right),
\end{aligned}
$$

and $X_{\mu \nu}^{(n)}$ are defined by Eqs. (3.5)-(3.7). The dimensionless coefficients $\tilde{\alpha}_{n}$ and $\tilde{\beta}_{n}$ are determined by the Horndeski action and are assumed to be order unity. $\Lambda_{3}$ is a parameter with the mass dimension, which decides the strong coupling scale. Note that, only in this appendix are the scalar field $\pi$ and the metric perturbation $h_{\mu \nu}$ normalized to be mass dimension one. When the $\mathcal{L}_{5}$ term in Horndeski theory is nonzero, the parameter $\tilde{\beta}_{3}$ is generally nonzero, which we assume here (see [17]).

We assume the existence of the Vainshtein screening solution in the spacetime region such as $\partial \partial h \gg \Lambda_{3}^{3}$; thus the metric perturbation is locally approximated by a solution of GR. We also assume the scalar field is split into some nonlinear expectation value and a fluctuation: $\pi=\bar{\pi}+\delta \pi$ with $\bar{\pi} \gg \delta \pi$. Then, the linearized Einstein equation is given by

$$
\mathcal{E}_{\mu \nu, \alpha \beta} h^{\alpha \beta}+\sum_{n=1}^{3} \frac{\tilde{\beta}_{n}}{\Lambda_{3}^{3(n-1)}} h^{\mu \nu} X_{\mu \nu}^{(n)}(\bar{\pi})=\frac{1}{M_{\mathrm{pl}}} T_{\mu \nu} .
$$

When the Vainshtein mechanism works (i.e., the metric perturbation is approximated by the solution of GR), the metric perturbation and the scalar field should satisfy the following inequalities: 


$$
\partial \partial h \gg \partial \partial \bar{\pi}, \quad \frac{1}{\Lambda_{3}^{3}}(\partial \partial \bar{\pi})^{2}, \quad \frac{1}{\Lambda_{3}^{6}}(\partial \partial \bar{\pi})^{3} .
$$

The quadratic Lagrangian for the scalar fluctuation is given by

$$
\mathcal{L}_{2}=-\frac{1}{2}\left(Z_{\text {gal }}^{\mu \nu}+Z_{h}^{\mu \nu}\right) \partial_{\mu} \delta \pi \partial_{\nu} \delta \pi
$$

where

$$
Z_{\text {gal }}^{\mu \nu}=\tilde{\alpha}_{2} \eta^{\mu \nu}+\sum_{n=1}^{3} \frac{\tilde{\alpha}_{n+2}}{\Lambda_{3}^{3 n}} X^{(n) \mu \nu}(\bar{\pi})
$$

$$
\begin{aligned}
Z_{h}^{\mu \nu}= & -\frac{M_{\mathrm{pl}}}{4 \Lambda_{3}^{3}} \epsilon^{\mu \alpha \beta \gamma} \epsilon^{\nu} \alpha^{\prime} \beta^{\prime} \gamma^{\prime} R^{(1) \alpha^{\prime} \beta^{\prime}}{ }_{\alpha \beta}(h) \\
& \times\left(\tilde{\beta}_{2} \delta^{\gamma^{\prime}}{ }_{\gamma}+\frac{\tilde{\beta}_{3}}{\Lambda_{3}^{3}} \bar{\Pi}^{\gamma^{\prime}}{ }_{\gamma}\right),
\end{aligned}
$$

with the linearized Riemann curvature $R^{(1) \alpha^{\prime} \beta^{\prime}}{ }_{\alpha \beta}(h) \sim$ $\partial \partial h / M_{\mathrm{pl}}$. The inequalities (A7) and $\partial \partial h \gg \Lambda_{3}^{3}$ suggest

$$
Z_{\text {gal }}^{\mu \nu} \ll Z_{h}^{\mu \nu},
$$

which means that the discussion in Sec. III can be applied to the case of the Horndeski theory and then the fluctuation $\delta \pi$ suffers from a ghost and/or a gradient instability.
[1] T. Clifton, P. G. Ferreira, A. Padilla, and C. Skordis, Phys. Rep. 513, 1 (2012).

[2] A. Joyce, B. Jain, J. Khoury, and M. Trodden, Phys. Rep. 568, 1 (2015).

[3] K. Koyama, Rep. Prog. Phys. 79, 046902 (2016).

[4] M. Fierz and W. Pauli, Proc. R. Soc. A 173, 211 (1939).

[5] H. van Dam and M. J. G. Veltman, Nucl. Phys. B22, 397 (1970).

[6] V. I. Zakharov, Pis'ma Zh. Eksp. Teor. Fiz. 12, 447 (1970) [JETP Lett. 12, 312 (1970)].

[7] A. I. Vainshtein, Phys. Lett. 39B, 393 (1972).

[8] A. Nicolis, R. Rattazzi, and E. Trincherini, Phys. Rev. D 79, 064036 (2009).

[9] C. Deffayet, G. Esposito-Farese, and A. Vikman, Phys. Rev. D 79, 084003 (2009).

[10] C. Deffayet, S. Deser, and G. Esposito-Farese, Phys. Rev. D 80, 064015 (2009).

[11] C. Deffayet, X. Gao, D. A. Steer, and G. Zahariade, Phys. Rev. D 84, 064039 (2011).

[12] T. Kobayashi, M. Yamaguchi, and J. Yokoyama, Prog. Theor. Phys. 126, 511 (2011).

[13] G. W. Horndeski, Int. J. Theor. Phys. 10, 363 (1974).

[14] J. Gleyzes, D. Langlois, F. Piazza, and F. Vernizzi, Phys. Rev. Lett. 114, 211101 (2015).

[15] J. Gleyzes, D. Langlois, F. Piazza, and F. Vernizzi, J. Cosmol. Astropart. Phys. 02 (2015) 018.

[16] R. Kimura, T. Kobayashi, and K. Yamamoto, Phys. Rev. D 85, 024023 (2012).

[17] K. Koyama, G. Niz, and G. Tasinato, Phys. Rev. D 88, 021502 (2013).

[18] T. Kobayashi, Y. Watanabe, and D. Yamauchi, Phys. Rev. D 91, 064013 (2015).

[19] R. Saito, D. Yamauchi, S. Mizuno, J. Gleyzes, and D. Langlois, J. Cosmol. Astropart. Phys. 06 (2015) 008.

[20] C. de Rham and G. Gabadadze, Phys. Rev. D 82, 044020 (2010).

[21] C. de Rham, G. Gabadadze, and A. J. Tolley, Phys. Rev. Lett. 106, 231101 (2011).
[22] S. F. Hassan and R. A. Rosen, J. High Energy Phys. 02 (2012) 126.

[23] K. Hinterbichler, Rev. Mod. Phys. 84, 671 (2012).

[24] E. Babichev and C. Deffayet, Classical Quantum Gravity 30, 184001 (2013).

[25] C. de Rham, Living Rev. Relativ. 17, 7 (2014).

[26] A. Schmidt-May and M. von Strauss, J. Phys. A 49, 183001 (2016).

[27] E. Babichev, C. Deffayet, and R. Ziour, J. High Energy Phys. 05 (2009) 098.

[28] E. Babichev, C. Deffayet, and R. Ziour, Phys. Rev. Lett. 103, 201102 (2009).

[29] E. Babichev, C. Deffayet, and R. Ziour, Phys. Rev. D 82, 104008 (2010).

[30] K. Koyama, G. Niz, and G. Tasinato, Phys. Rev. Lett. 107, 131101 (2011).

[31] T. M. Nieuwenhuizen, Phys. Rev. D 84, 024038 (2011).

[32] K. Koyama, G. Niz, and G. Tasinato, Phys. Rev. D 84, 064033 (2011).

[33] G. Chkareuli and D. Pirtskhalava, Phys. Lett. B 713, 99 (2012).

[34] A. Gruzinov and M. Mirbabayi, Phys. Rev. D 84, 124019 (2011).

[35] E. Babichev, C. Deffayet, and G. Esposito-Farese, Phys. Rev. Lett. 107, 251102 (2011).

[36] D. Comelli, M. Crisostomi, F. Nesti, and L. Pilo, Phys. Rev. D 85, 024044 (2012).

[37] L. Berezhiani, G. Chkareuli, C. de Rham, G. Gabadadze, and A. J. Tolley, Phys. Rev. D 85, 044024 (2012).

[38] S. Sjors and E. Mortsell, J. High Energy Phys. 02 (2013) 080.

[39] M. S. Volkov, Phys. Rev. D 85, 124043 (2012).

[40] F. Sbisa, G. Niz, K. Koyama, and G. Tasinato, Phys. Rev. D 86, 024033 (2012).

[41] M.S. Volkov, Classical Quantum Gravity 30, 184009 (2013).

[42] E. Babichev and M. Crisostomi, Phys. Rev. D 88, 084002 (2013). 
[43] N. Kaloper, A. Padilla, P. Saffin, and D. Stefanyszyn, Phys. Rev. D 91, 045017 (2015).

[44] S. Renaux-Petel, J. Cosmol. Astropart. Phys. 03 (2014) 043.

[45] J. Enander and E. Mortsell, J. Cosmol. Astropart. Phys. 11 (2015) 023.

[46] K. Aoki, K.-i. Maeda, and R. Namba, Phys. Rev. D 92, 044054 (2015).

[47] K. Aoki, K.-i. Maeda, and M. Tanabe, Phys. Rev. D 93, 064054 (2016).

[48] N. Arkani-Hamed, H. Georgi, and M. D. Schwartz, Ann. Phys. (Amsterdam) 305, 96 (2003).

[49] N. A. Ondo and A. J. Tolley, J. High Energy Phys. 11 (2013) 059.

[50] M. Fasiello and A. J. Tolley, J. Cosmol. Astropart. Phys. 12 (2013) 002.

[51] C. de Rham, A. J. Tolley, and S.-Y. Zhou, Phys. Lett. B 760, 579 (2016).
[52] C. de Rham, A. J. Tolley, and S.-Y. Zhou, J. High Energy Phys. 04 (2016) 188.

[53] V. Baccetti, P. Martin-Moruno, and M. Visser, Classical Quantum Gravity 30, 015004 (2013).

[54] E. Babichev and A. Fabbri, Classical Quantum Gravity 30, 152001 (2013).

[55] R. Brito, V. Cardoso, and P. Pani, Phys. Rev. D 88, 023514 (2013).

[56] H. Kodama and I. Arraut, Prog. Theor. Exp. Phys. 2014, 23E02 (2014).

[57] E. Babichev and A. Fabbri, Phys. Rev. D 89, 081502 (2014).

[58] E. Babichev, R. Brito, and P. Pani, Phys. Rev. D 93, 044041 (2016).

[59] E. Babichev and R. Brito, Classical Quantum Gravity 32, 154001 (2015).

[60] R. Brito, V. Cardoso, and P. Pani, Phys. Rev. D 88, 064006 (2013). 